\title{
Cardiac Involvement in Total Generalized Lipodystrophy (Berardinelli- Seip Syndrome)
}

\author{
Ruy Felipe Melo Viégas, Rosiane Viana Zuza Diniz, Taciana Mara Rezende Fortes Viégas, \\ Edgar Bezerra Lira Fo, Dirceu Rodrigues de Almeida
}

São Paulo, SP - Brazil

\begin{abstract}
Total generalized lipodystrophy (Berardinelli-Seip Syndrome) is a rare hereditary disease characterized by insulin-resistant diabetes mellitus and a small quantity of adipose tissue and is of unknown origin. Common cardiovascular alterations related to this syndrome are cardiac hypertrophy and arterial hypertension. This article reports a case of Berardinelli-Seip syndrome and reviews the literature with special emphasis on the cardiovascular manifestations of this syndrome.
\end{abstract}

Total generalized lipodystrophy, also known as Berardinelli-Seip syndrome, is a rare hereditary syndrome of indeterminate cause characterized by severe diabetes mellitus, no ketosis/ketonuria, insulin resistance, and by the almost total lack of subcutaneous adipose tissue ${ }^{1,2}$. The biochemical anomalies found in this syndrome have, as a basic mechanism, an abnormal response to insulin action and the incapability of producing adipose tissue ${ }^{2}$. Other characteristics include gum hypertrophy, hepatomegaly affecting liver functions, hypertriglyceridemia, acanthosis nigricans, arterial hypertension, hirsutism, nephropathy, and cardiac hypertrophy ${ }^{3-6}$. Although this syndrome was described 40 years ago, little has been reported about its cardiovascular manifestations and its prognosis. In this study, we report a case of total generalized lipodystrophy, accompanied by review of the literature, and an analysis of the cardiovascular manifestations of this anomaly.

\section{Case Report}

The patient is a woman with type I diabetes mellitus and severe insulin resistance, diagnosed four years earlier, who requires high doses of NPH insulin for clinical compensation (3UI/kg weight). Since infancy, the patient has had a history of weight loss, gigantism, and intellectual under-

Universidade Federal de São Paulo - Escola Paulista de Medicina

Correspondência: Ruy Felipe Melo Viégas - UNIFESP-EPM - Casa de Acompanhamento do Cardiopata - Rua dos Otonis, 897 - 04025-002 - São Paulo, SP - Brazil development. She was admitted to the hospital with progressive breathlessness during stress, which evolved to breathlessness at rest and orthopnea plus edema of the lower limbs. On clinical examination the patient weighed $40 \mathrm{~kg}$, was $1.70 \mathrm{~m}$ tall, with a body mass index of $13.8 \mathrm{~kg} / \mathrm{m}^{2}$, a thin general appearance, atrophy of the subcutaneous cellular tissue, acanthosis nigricans in the cervical region, gum hypertrophy, and hirsutism on the lower limbs (fig.1.) When examined with cardiovascular apparatus, her blood pressure was $140 / 100 \mathrm{mmHg}$, and her heart rate was $110 \mathrm{bpm}$. She had absence of jugular turgescence, normal cardiac ictus and bulla with a murmur of systolic ejection $++/ 4+$ in the left medium external border. When palpating the abdomen, the liver was found to be $2 \mathrm{~cm}$ from the right costal edge. Inferior limbs had edema $+/ 4+$. The electrocardiogram showed sinus rhythm, a heart rate of $110 \mathrm{bpm}, \mathrm{SAQRS}$ of + $60^{\circ}$, PR interval 0.12s, and QT interval 0.22s. The electrocardiogram had inverted, flattened waves and symmetry in the precordial leads $\mathrm{V}_{4}, \mathrm{~V}_{5}, \mathrm{~V}_{6}$ and in $\mathrm{D}_{2}, \mathrm{D}_{3}$, and AVF. The chest X-ray showed a normal cardiac silhouette with important, bilateral pulmonary congestion (fig. 2). The transthoracic echocardiogram showed normal cardiac chamber anatomy, valves and great vases with severe symmetric hypertrophy and hyperdynamic left ventricle performance, with the interventricular septum measuring $16 \mathrm{~mm}$ and $\mathrm{mass} / \mathrm{superficies}$ relation, $236.17 \mathrm{~g} / \mathrm{m}^{2}$ and with Doppler, a restrictive pattern to mitral flow (fig. 3). Magnetic resonance imaging showed the heart in the usual position, with intense thickening of the left ventricle wall and the interventricular septum with limited diastolic volume (fig. 4 and 5). Abdominal ultrasound showed homogenous hepatosplenomegaly and bilateral parenchymal nephropathy. Bioimpedance showed a presence of $1 \%$ of total body fat (normal value -20 to 26 ), in relation to thin mass/ fat, 66.4:1 (ideal value 2.8:1 or greater). The results of laboratory examinations were: creatinine, $0.9 \mathrm{mg} / \mathrm{dL}$; urea, 19mg/dL; TGO, 16UI/1; TGP, 23 UI/1; TAP, 100\%; 'GAMA GT, $65 \mathrm{UI} / 1$; alkaline phosphate, $224 \mathrm{UI} / 1$; hemoglobin, $7.7 \mathrm{~g} / \mathrm{dL}$; hematocrit, $23 \%$; triglycerides, $644 \mathrm{mg} / \mathrm{dL}$; total cholesterol, 119mg/dL; cholesterol HDL, 26mg/dL; and fasting glycemia, 222mg/dL. Fundoscopy showed light to medium proliferating (spreading) diabetic retinopathy and 


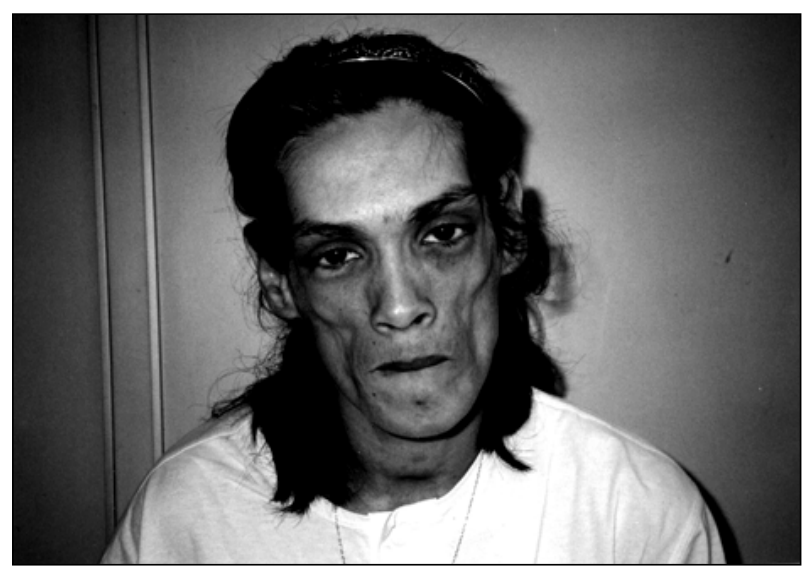

Fig. 1 - Phenotype in total generalized lipodystrophy.

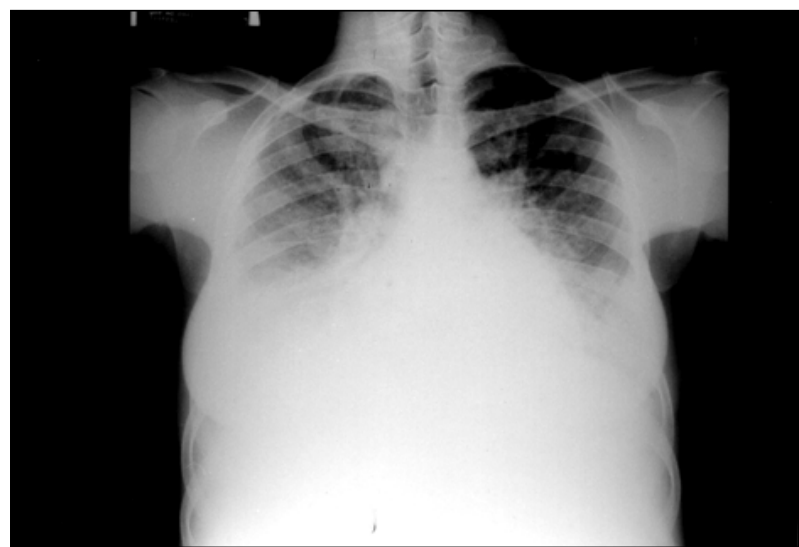

Fig. 2 - Chest X-Ray showing pulmonary congestion in the presence of normal sized cardiac shadow.

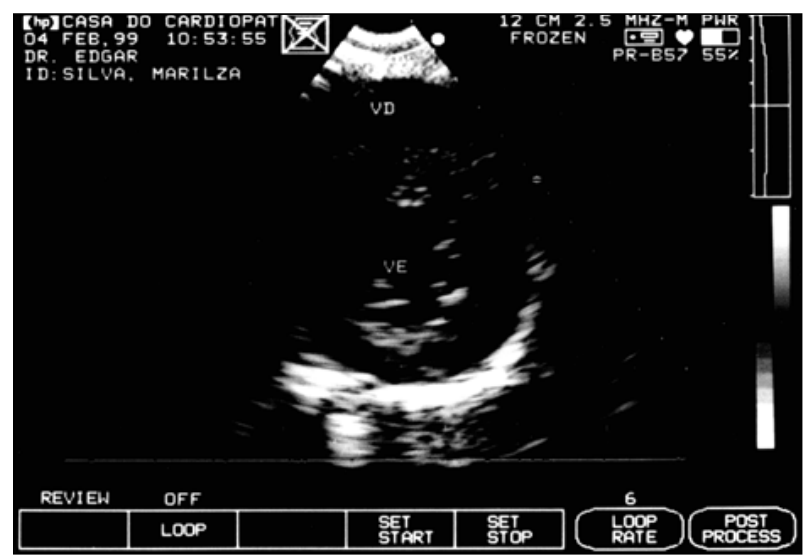

Fig. 3 - Significant symmetrical myocardial hypertrophy demonstrated by echocardiography.

light hypertensive retinopathy. Treatment began with loop diuretics and calcium channel-blockers (verapamil) with clinical improvement within 3 days. She was discharged in functional class I.

Cardiac involvement in total generalized lipodystrophy has not been reported in the literature. After a review, we found only 17 reported cases.
Cardiac involvement in total generalized lipodystrophy
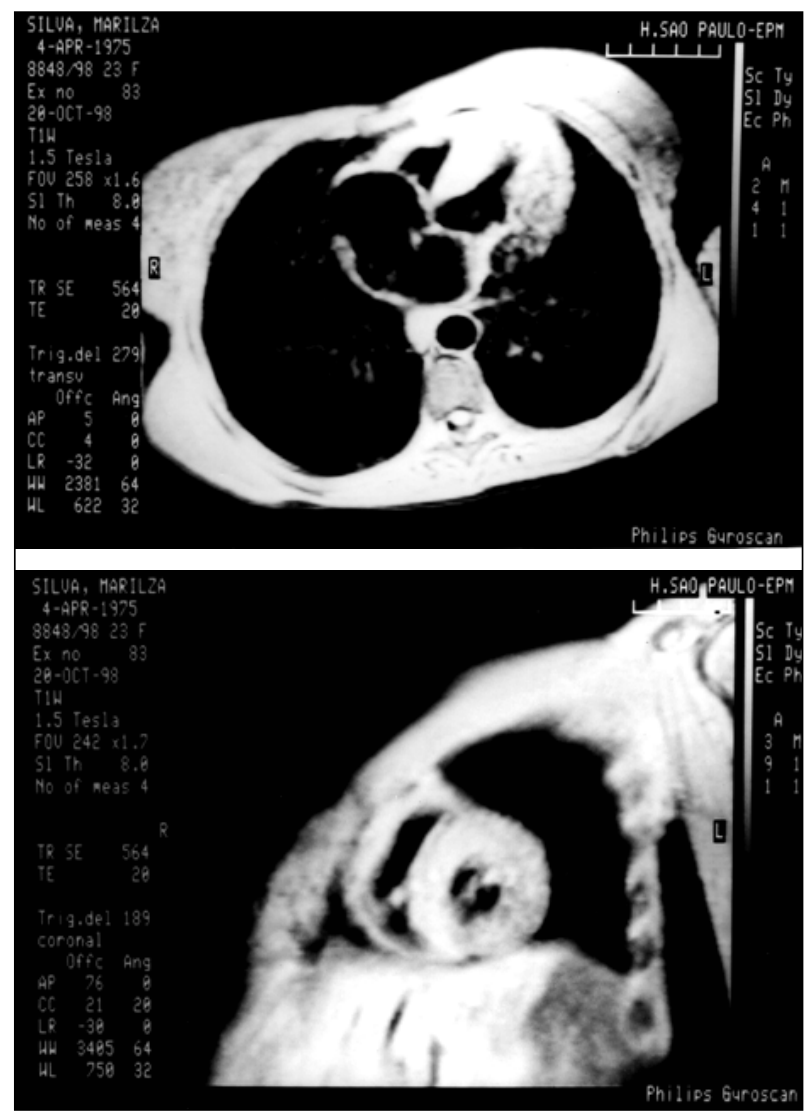

Fig. 4 and fig. 5 - Nuclear magnetic resonance imaging of myocardial hypertrophy.

Bjornstad el al ${ }^{4,7}$ described the evolution of seven patients with congenital generalized lipodystrophy, two women and five men, aged between 9-29, average 20.5 age, all with light systolic murmur at the left sternal edge, five with symptoms of left ventricular overload on electrocardiogram, and all with discreet cardiomegaly. In the two-dimensional echocardiography, myocardial hypertrophy was observed in all patients, symmetric in four and asymmetric (septum predomination) in two patients. The shortening fraction was normal in all cases. On evolution, four patients died at an average age of 32 years.

Rheuban et al ${ }^{5}$ described four cases of total generalize lipodystrophy (16-23 years, average age 19 years) all presenting with systolic murmur in the apex, with light arterial hypertension in two patients. On echocardiographic evaluation, all patients had symmetric hypertrophic cardiomyopathy, and normal systolic function. During necropsy of one patient, the histologic finding indicated accentuated hypertrophy of fibers, without rearrangement of the fibers and without glycogenic deposits. Geffner et $\mathrm{al}^{8}$ report the case of a six-month-old child with severe insulin resistance and severe myocardial hypertrophy. Klar et $\mathrm{al}^{6}$ report the case of a patient, a 13-year-old girl, with severe insulin resistance, and nonobstructive asymmetric septal hypertrophy, observed on echocardiography, with normal systolic function. 


\section{Discussion}

The case presented is a report of total generalized lipodystrophy in which the patient presented with a thin general appearance due to atrophy of subcutaneous cellular tissue. The boimpedance examination confirmed this, indicating the total presence of $1 \%$ of fat, a severe deficit, and a common occurrence in cases of lipodystrophy reported in literature ${ }^{1,2}$.

Hepatomegaly ${ }^{4-6,8}$ is a frequent finding and was also found in our patient, in spite of her well-preserved liver function, which is contrary to findings by Bjornstad ${ }^{4}$. Hypertriglyceridemia has been reported ${ }^{3-6}$.

Acanthosis nigricans, which is found in diabetic patients who react with hyperinsulinemia and peripheral resistance to insulin, was also present on clinical examination.

In Bjornstad's ${ }^{7}$ study, myocardial hypertrophy and ventricular diastolic dysfunction were found in greater frequency in patients with lipodystrophy, related to the progressive increase in age, facts found in our patient in whom hypertrophy was considered severe. The clinical and echocardiographic findings and the quick clinical improvement in our patient are indications that the diastolic dysfunction was responsible for the exuberant status of cardiac failure observed in this patient. The level I hypertension found does not seem to be the cause of myocardial hypertrophy. Rheuban et al ${ }^{5}$ found normal tension levels in half of the patients with lipodystrophy with hypertrophic myocardiopathy.

Hypertrophic cardiomyopathy, which occurs in patients with genetic insulin resistance syndrome, must be an expression of the insulin action in the heart for its action in the cardiac receptors of Insulin-Like growth factor I (IGF-1) ${ }^{3}$. This is strongly based on the documented frequent occurrence of myocardial hypertrophy in children of diabetic mothers and in patients with Beckwith-Wiedemann ${ }^{6}$ syndrome.

Two hormones are important in the understanding of the physiopathology of this syndrome: insulin and IGF-1, which have in common their own receptors distributed in certain organs and tissues, with similar function and structure, that when activated promote growth and cellular differentiation. The activation of these receivers determines all the clinical and biological responses produced by the link with its hormones ${ }^{3}$. The excess of a hormone may activate the receiver of another hormone. When a receptor is activated by a hormone that is not its homologue, the event is called specificity spillover ${ }^{6}$. In patients who are in an insulin-resistant state and therefore, with hyperinsulinemia, the linking of insulin to the IGF- $1{ }^{3}$ receptors present in the myocardial tissues results in hypertrophy of this organ, as well as other tissues where these receptors are distributed ${ }^{3,5,8}$. This explains the high frequency of myocardial hypertrophy in some cases of insulin resistance, like in lipodystrophy ${ }^{6,7}$. The histological differentiation from the classic hypertrophic cardiomyopathy is made by the absence of rearrangement of fibers in the myocardial hypertrophy associated with lipodystrophy.

In conclusion, cardiac involvement seems to be the bigger influence in the long-term prognosis of patients with the syndrome ${ }^{7}$, with an unfavorable characteristic due to the anatomic and functional changes that occur as a result of the severe ${ }^{3}$ myocardial hypertrophy.

\section{References}

1. Berardinelli W. An undiagnosed endocrinometabolic syndrome: report of 2 cases. J Clin Endocrin Metab 1954; 14: 193-204.

2. Selp M. Lypodystrophy and gigantism with associated endocrine manifestation. Acta Paediatr 1959; 48: 555-74

3. Geffner ME, Golde DW. Selective insulin action on skin, ovary, and heart in insulin-resistant states. Diabetes Care 1988; 11: 500-05.

4. Bjornstad PG, Semb BKH, Trygstad O, Seip M. Echocardiographic assessment of cardiac function and morphology in patients with generalised lipodystrphy. Eur J Pediatr 1985; 144: 355-9.

5. Rheuban KS, Blizzard RM, Parker M, Carter T, Wilson T, Gutgesell HP.
Hypertrophic cardiomyopathy in total lipodystrophy. J Pediatr 1986; 109: 301-2

6. Klar A, Brand A, Hurwitz H, Gross-Kieselstein E, Branski D. Cardiomyopathy in lypodystrophy and the specificity of the spillover hypothesis. Isr J Med Sci 1993; 29: 50-2.

7. Bjornstad PG, Foerster A, İhlen H. Cardiac findings in generalized lipodystrophy. Acta Paediatr Suppl 1996; 413: 39-43.

8. Geffner ME, Santulli TV, Kaplan AS. Hypertrophic cardiomyopathy in total lipodystrophy: Insulin action in the face of insulin resistance? J Pediatr 1987; 110: 161. 